\title{
ISAR: An Interactive System for Algebraic Implementation Proofs
}

\author{
Bernhard Bauer, Rolf Hennicker \\ Inst. für Informatik, Ludwig-Maximilians Üniversität München, Leopoldstr. 11 b, D-8000 München
}

\section{Basic concepts}

Formal implementation notions are a necessary prerequisite for proving the correctness of software development steps. In order to be useful in practice formal implementation concepts should be supplied by appropriate proof methods and, even more important, by tools providing mechanical support for correctness proofs. In the following an interactive system, called ISAR, is described which provides an environment for proofs of algebraic implementation relations based on behavioural semantics of equational algebraic specifications. For the basic notions of algebraic specifications, such as signature $\Sigma$, term algebra $\mathrm{W}_{\Sigma}(\mathrm{X})$, ground term algebra $\mathrm{W}_{\Sigma}$ etc. we refer to [2]. Then a behavioural specification $\mathrm{SP}=$ $(\Sigma$, Obs, E) consists of a signature $\Sigma=(S, F)$, a subset Obs $\subseteq S$ of observable sorts and a set $\mathrm{E}$ of axioms (here equations $\mathrm{t}=\mathrm{r}$ with terms $\mathrm{t}, \mathrm{r} \in \mathrm{W}_{\Sigma}(\mathrm{X})$ ).

The definition of our implementation concept is based on the assumption that from the software user's point of view a software product is a correct implementation if it satisfies the desired input/output behaviour. Hence a behavioural specification SP1 $=(\Sigma 1$, Obs1, $\mathrm{E} 1)$ is called behavioural implementation of $\mathrm{SP}=(\Sigma, \mathrm{Obs}, \mathrm{E})$ if SP1 respects the observable properties of SP. A precise formal definition of this intuitive notion on the model level is given in [4]. Since we are interested in automatic implementation proofs we will present here only the following proof theoretic characterization for behavioural implementations (cf. [4]) which is the theoretical basis of the ISAR-system. The characterization uses the notion of observable $\Sigma$-context which is any term $c\left[z_{S}\right]$ of observable sort $s_{0} \in$ Obs over the signature $\Sigma$ of SP which contains a distinguished variable $z_{S}$ of some sort $s \in S$. The application of a context $c\left[z_{S}\right]$ to a term $t$ of sort $s$ is defined by the substitution of $z_{S}$ by $t$. It is denoted by $c[t]$.

\section{Proof theoretic characterization of behavioural implementations}

Let $\mathrm{SP} 1=(\Sigma 1, \mathrm{Obs} 1, \mathrm{E} 1)$ and $\mathrm{SP}=(\Sigma, \mathrm{Obs}, \mathrm{E})$ be behavioural specifications such that $\Sigma \subseteq \Sigma 1$ and Obs $\subseteq$ Obs1. SP1 is a behavioural implementation of SP if and only if for all observable $\Sigma$-contexts $\mathrm{c}\left[\mathrm{z}_{\mathrm{S}}\right]$ the following property $\mathrm{P}\left(\mathrm{c}\left[\mathrm{z}_{\mathrm{S}}\right]\right)$ is valid:

$\mathrm{P}\left(\mathrm{c}\left[\mathrm{z}_{\mathrm{S}}\right]\right)=$ true $\Leftrightarrow$ for all axioms $(\mathrm{t}=\mathrm{r}) \in \mathrm{E}$ and for all ground substitutions $\sigma: X \rightarrow W_{\Sigma}$ the following holds: if $t$ is of sort $s$ then $E 11-\sigma(c[t])=\sigma(c[r])$.

The usual subterm ordering defines a Noetherian relation on the set of observable $\Sigma$-contexts and therefore we can use structural induction for showing the validity of $P\left(c\left[z_{S}\right]\right)$ for all observable $\Sigma$-contexts $\mathrm{c}\left[\mathrm{z}_{\mathrm{S}}\right]$. The automatization of such induction proofs over the structure of contexts (also called context induction) is the basic principle of the ISAR-system.

\section{Proof of Correct Implementation Steps by the ISAR-System}

The input of the ISAR-system is the description of an implementation step consisting of three parts: an abstract specification $S P=(\Sigma$, Obs, E) to be implemented, a concrete specification SP-C $=(\Sigma-C$, Obs-C, E-C $)$ used as a basis for the implementation and a construction of the implementation. The implementation construction can be defined by some enrichment and/or renaming of SP-C. For instance the following implementation step performs first a renaming of SP-C w.r.t. a signature morphism $\sigma: \Sigma-C \rightarrow \Sigma$ and then an enrichment $\Delta=\left\langle\Sigma^{\prime}\right.$, Obs', $\left.E^{\prime}\right\rangle$ of the renamed version of SP-C:

implementation step SP_by_SP-C = SP is implemented by SP-C $v i a$ renaming $\sigma$, enrichment $\Delta$ endimpl step 
Such an implementation step is called correct if the normalization of enrich (rename SP-C by $\sigma)$ by $\Delta==_{\text {def }}\left(\sigma(\Sigma-C) \cup \Sigma, \sigma(O b s-C) \cup O b s^{4}, \sigma(E-C) \cup E^{\prime}\right)$ is a behavioural implementation of SP. The correctness of implementation steps when performing first an enrichment and then a renaming can be defined analogously.

The normalization of the construction of the implementation is computed by the normalizer module of the ISAR-system (cf. section 3). Since the normalizer can also be used for flattening arbitrary behavioural specifications which are structured by enrichment, renaming and even combination of specifications the abstract specification SP and the concrete specification SP-C can be structured specifications as well. As an example we consider an implementation step which implements a specification SET of finite sets over arbitrary elements on top of a specification LIST of finite lists of elements. For more complicated non-standard examples which, for lack of space, cannot presented here we refer to [1] where e.g. the implementation of stacks by arrays with pointers is proved (using an auxiliary function iterated-pop which defines an iteration of pop operations) and the proof of an implementation of an abstract specification of an imperative programming language by a state-oriented specification of the language is demonstrated. (In the following the variables occurring in the axioms are generated and typed by the system if an implementation step is displayed.)

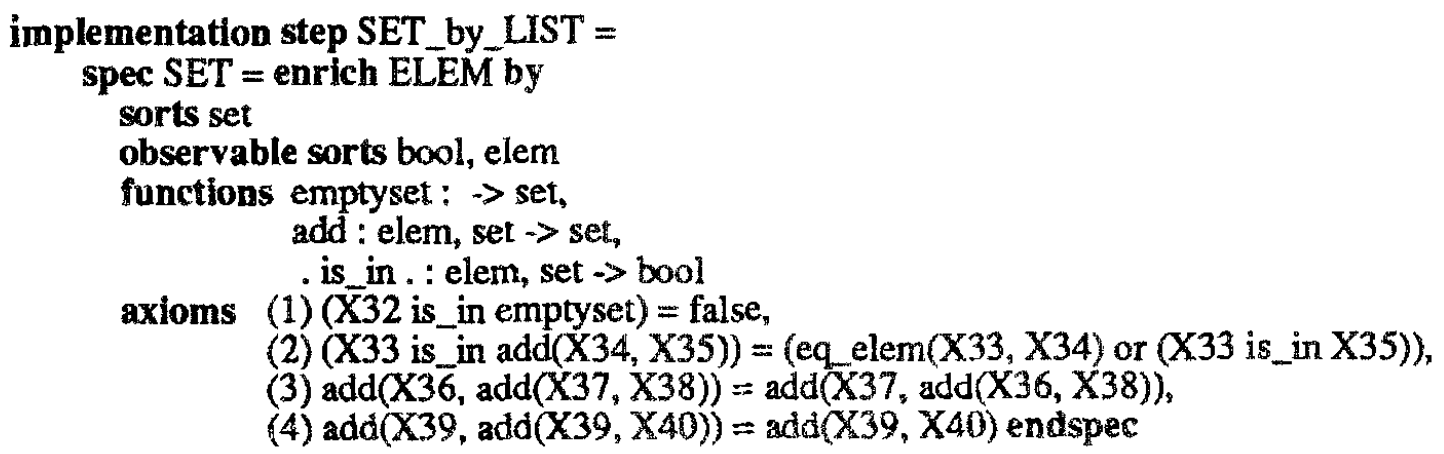

is implemented by

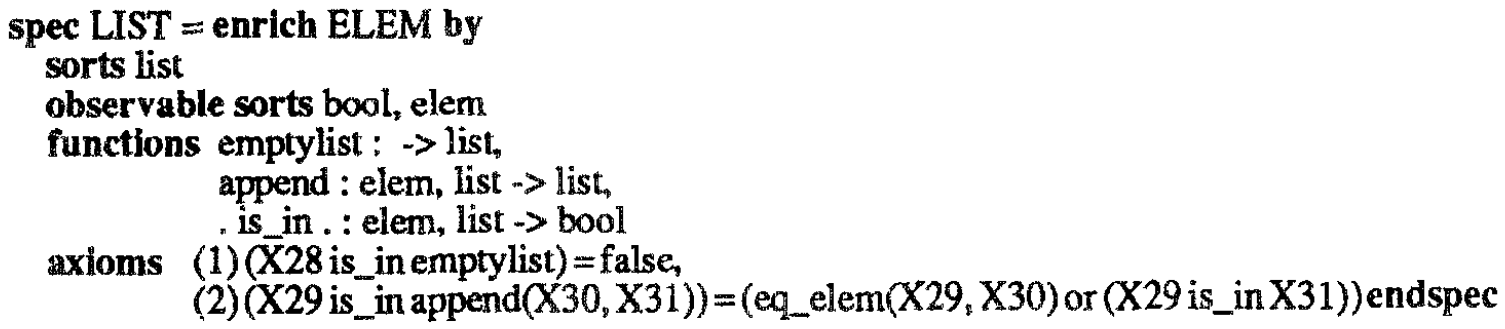

via renaming [set/list], enrichment functions emptyset : $\rightarrow$ set, add : elem, set $\rightarrow$ set axioms emptyset $=$ emptylist, $\operatorname{add}(\mathrm{X} 41, \mathrm{X} 42)=$ append $(\mathrm{X} 41, \mathrm{X} 42)$ endimplstep

Now the correctness of the implementation step SET by LIST can be proved by the ISARsystem using the principle of context induction. The underlying algorithm of the context induction prover of the ISAR-system is described in [5]. For lack of space we do not present here the complete session of the implementation proof but we show the summary of the proof which can be displayed at the end of the session. Thereby any equation occurring as a proof obligation which has been deduced from the axioms of the implementing specification is marked by the comment "* proved". For the proof of equations the ISAR-system is connected to the TIP-system which is an inductive theorem prover (cf. [3]). 
* DEPTH: 0

* PROOF OBLIGATIONS:

X32 is_in emptyset $=$ false * proved

$X 33$ is_in add $(X 34, X 35)=$ eq_elem $(X 33, X 34)$ or X33 is_in X35* proved

*** CONTEXT INDUCTION STEP FOR CONTEXTS OF SORTS: bool, elem

Selected function: . is in .: elem, set $\rightarrow$ bool

* SELECTED CONTEXT: X84 is_in $z$ _set

*** BASE OF THE CONTEXT INDUCTION FOR CONTEXTS OF SORT: set

* DEPTH: 1

* PROOF OBLIGATIONS:

$X 84$ is_in add $(X 36, \operatorname{add}(X 37, X 38))=X 84$ is_in $\operatorname{add}(X 37, \operatorname{add}(X 36, X 38)) *$ proved

$\mathrm{X} 84$ is in add(X39, add $(\mathrm{X} 39, \mathrm{X} 40))=\mathrm{X} 84$ is in add $(\mathrm{X} 39, \mathrm{X} 40))^{*}$ proved

** CONTEXT INDUCTION STEP FOR CONTEXTS OF SORT: set

Selected function: add : elem, set $\rightarrow$ set

- ADDITIONAL HYPOTHESIS OF THE CONTEXT-INDUCTION:

$X 84$ is_in constant1_set $=X 84$ is_in constant2_set

PROOF OBLIGATIONS:

$X 84$ is_in add $(X 363$, constant1_set $)=X 84$ is_in add(X363, constant2_set) ${ }^{*}$ proved

* END OF THE IMPLEMENTATION PROOF: ALL PROOF OB̈LIGATIONS PROVED *

\section{The Structure of the ISAR-System}

The ISAR-system is connected to the TIP-system which verifies all proof obligations generated by the ISAR-system. The main modules of the ISAR-system are

- a scanner and parser with a mixfix-parser for the syntactical analysis of the specifications and implementation steps,

- a normalizer for flattening structured specifications,

- a context induction prover which is the heart of the system for proving the correctness of implementation steps,

- a context generator to produce automatically contexts for context induction steps (the selection of an appropriate context which is general enough for successful termination of implementation proofs has then to be done by the user),

- a TIP-interface for the exchange of informations between the ISAR and the TIP-system

- the proof-modules of the TIP-system for the verification of the proof obligations.

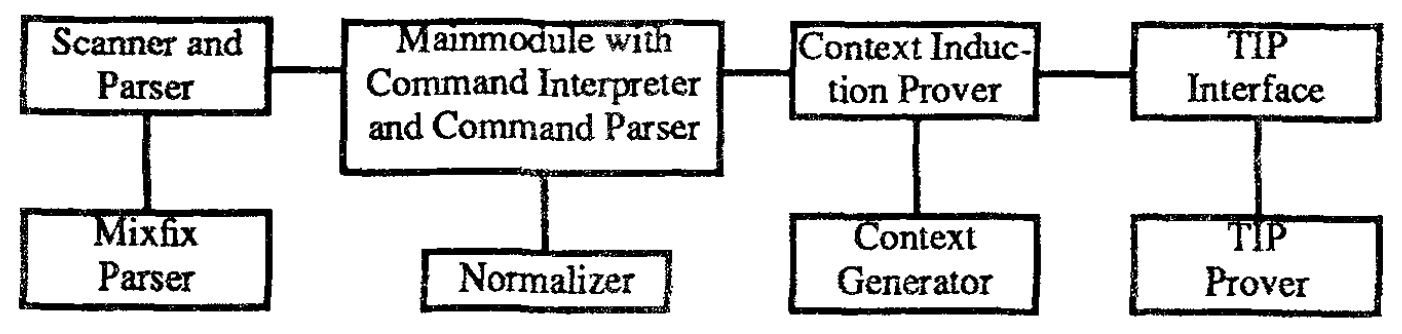

\section{References}

1. B. Bauer: Ein interaktives System für algebraische Implementierungsbeweise. Diplomarbeit, Fakultät für Mathematik und Informatik, Universität Passau, 1992.

2. H. Ehrig, B. Mahr, Fundamentals of algebraic specification 1, EATCS Monographs on Theor. Comp. Science 6, Springer, Berlin, 1985.

3. U. Fraus, H. Hußmann: An inductive theorem prover based on narrowing. Proc. LPAR '92, Logic Programming and Automated Reasoning, Lecture Notes in Artificial Intelligence, Springer, 1992.

4. R. Hennicker: Context induction: a proof principle for behavioural abstractions and algebraic implementations. Formal Aspects of Computing, 3 (4), 1991.

5. R. Hennicker: A semi-algorithm for algebraic implementation proofs. Technical Report MIP. 9108, Univ. Passau, 1991. Ext. version to appear in: Theoretical Computer Science, 104, 1993. 